\title{
Obesity and visceral fat: a growing inflammatory disease
}

\author{
Stephen B Hanauer
}

Developed nations have replaced malnutrition and infectious diseases as causes of increased morbidity and mortality in childhood with obesity and its metabolic (and now inflammatory) consequences in adults. It is estimated that over $60 \%$ of adult Americans are obese or overweight, and nearly $25 \%$ of adult Americans have the 'metabolic syndrome'-a constellation of insulin resistance, visceral adiposity, hypertension and dyslipidemia. The multiple negative health consequences of overnutrition are gradually being unraveled: first, from epidemiologic observations regarding the risks of coronary artery disease and malignancies, and, subsequently, from mechanistic studies pertaining to the numerous sequelae of the metabolic syndrome.

A Review in this month's edition of Nature Clinical Practice Gastroenterology \& Hepatology from Schäffler and colleagues provides an extension of a previous Review on the role of mesenteric fat (visceral adipose tissue) in inflammatory bowel disease, ${ }^{1}$ to include its role in nonalcoholic fatty liver disease, steatosis and nonalcoholic steatohepatitis. There is increasing evidence that visceral adipose tissue is a metabolic and inflammatory organ in its own right. The metabolic syndrome also includes an increased risk of microalbuminemia, endothelial dysfunction, and a proinflammatory and prothrombotic state with elevated levels of C-reactive protein, tumor-necrosis factor- $\alpha$ and interleukin-6.

Visceral adipose tissue can no longer be considered simply as a passive fat-storage site, but as an endocrine organ that signals to and modulates the action and metabolism of the brain, liver, muscle, and vascular endothelial cells. Indeed, adipocytes have receptors for numerous peptides, steroids, and other hormones, catecholamines, and the cytokines leptin, interleukin-6 and tumor-necrosis factor- $\alpha$. Thus, while it has long been recognized that the endocrine system and immune system interact via the hypothalamic-pituitaryadrenal axis and the production of cortisone (a physiologic and pharmacologic mediator
We are moving

towards an

expanded

appreciation

of endocrine/

immune/

inflammatory

interactions

that provide

therapeutic

(pharmacologic)

targets for

modulation

of the

inflammatory

sequelae of

an expanded

adipocyte organ

system

SB Hanauer is Editorin-Chief of Nature Clinical Practice Gastroenterology \& Hepatology.

Competing interests

The author declared competing interests; go to

the article online for details.

www.nature.com/clinicalpractice doi:10.1038/ncpgasthep0197 of inflammation), our expanding knowledge regarding visceral adipose tissue augments our concept of endocrine/immune/inflammatory regulation and interactions.

Approaches to treat sequelae of obesity and the metabolic syndrome have included the use of statins to counter its dyslipidemic effects (and coronary risks) and glitazones to improve the insulin resistance seen in type II diabetes. The observation that statins have an independent impact on the levels of C-reactive protein, as well as reducing LDL, 2,3 provides further insight into the role of inflammatory cytokines derived from visceral adipose tissue as targets for the treatment or prevention of coronary artery disease (and perhaps other inflammatory sequelae related to obesity). Similarly, the glitazone agents used to reduce blood glucose levels in patients with diabetes have been shown to have anti-inflammatory properties related to the activation of peroxisome proliferator-activated receptors (ligand-activated transcription factors), including the inhibition of leukocyte infiltration into tissues, mediated by nuclear-factor-kB-dependent expression of endothelial-cell adhesion molecule. ${ }^{4}$

We are now beginning to recognize that mechanisms relating the effects of expansion of visceral adipose tissue to the metabolic syndrome, once thought to be metabolic, must be antiinflammatory. Consequently, the mechanisms underlying therapies for the sequelae of obesity and the metabolic syndrome, must also be antiinflammatory. Ultimately, prevention of obesity is the goal for avoiding the numerous downstream effects of the metabolic syndrome. Until that time, we are moving towards an expanded appreciation of endocrine/immune/inflammatory interactions that provide therapeutic (pharmacologic) targets for modulation of the inflammatory sequelae of an expanded adipocyte organ system.

Supplementary information, in the form of a reference list, is available on the Nature Clinical Practice Gastroenterology \& Hepatology website. 